\title{
PERIODIC MOTION IN NON-AXIALLY SYMMETRIC GALAXIES
}

\author{
JAUME LLIBRE ${ }^{1}$ AND CLAUDIO VIDAL ${ }^{2}$
}

\begin{abstract}
We apply the averaging theory for proving the existence of twelve families of periodic orbits in a three-dimensional galactic Hamiltonian systems.
\end{abstract}

\section{INTRODUCTION AND STATEMENTS OF MAIN RESUltS}

In order to study the dynamics of the galaxies we need to have a model describing the properties of their motion. Of course the information for obtaining the dynamical models of the motion of the galaxies comes from observations. In order to simplify the dynamics of their orbits these galactic models usually are axially symmetric or spherical. Spherical models for galaxies were studied in $[8,12,18]$. Moreover, interesting axially symmetric galaxy models were presented and studied in $[1,3,4,7,10,15,16,17,19]$.

The potential of our model is

$$
V(X, Y, Z)=-\frac{1}{\sqrt{X^{2}+Y^{2}+\left(a+\sqrt{b^{2}+Z^{2}}\right)^{2}}}+\frac{1}{a+b}, .
$$

In this potential the gravitation constant and the mass of the galaxy is one taking conveniently the unit of mass and of the distance, while $a$ and $b$ are parameters connected to the geometry of the galaxy. When and $b \gg a$ the model describes an elliptical galaxy with $a$ and $b$ being the scale-lengths of its semiaxes. See for more details about this potential the articles $[5,6,11,20]$. The main reason for choosing this potential is that despite of the major part of the galaxies are not exactly axially symmetric, the axial symmetry is a good approximation which simplifies the computations for studying the motion of the galaxy.

Our main goal is to study analytically the periodic motion of the Hamiltonian system associated to the Hamiltonian

$$
H=\frac{1}{2}\left(p_{X}^{2}+p_{Y}^{2}+p_{Z}^{2}\right)-\frac{1}{\sqrt{X^{2}+Y^{2}+\left(a+\sqrt{b^{2}+Z^{2}}\right)^{2}}},
$$

where $a>0$ and $b>0$. We omit the term of the potential $1 /(a+b)$ because it does not play any role in the motion.

2000 Mathematics Subject Classification. Primary 34C10, 34C25.

Key words and phrases. periodic orbits, galactic bar model, averaging theory. 
The Hamiltonian system associated to the Hamiltonian (1) is

$$
\begin{aligned}
\dot{X} & =p_{X}, \\
\dot{Y} & =p_{Y}, \\
\dot{Z} & =p_{Z} \\
\dot{p_{X}} & =-\frac{X}{\left[X^{2}+Y^{2}+\left(a+\sqrt{b^{2}+Z^{2}}\right)^{2}\right]^{3 / 2}}, \\
\dot{p}_{Y} & =-\frac{Y}{\left[X^{2}+Y^{2}+\left(a+\sqrt{b^{2}+Z^{2}}\right)^{2}\right]^{3 / 2}}, \\
\dot{p_{Z}} & =-\frac{Z\left(a+\sqrt{b^{2}+Z^{2}}\right)^{2}}{\sqrt{b^{2}+Z^{2}}\left[X^{2}+Y^{2}+\left(a+\sqrt{b^{2}+Z^{2}}\right)^{2}\right]^{3 / 2}} .
\end{aligned}
$$

As usual the dot denotes derivative with respect to the independent variable $t \in \mathbb{R}$, the time.

In general the periodic orbits are the most simple orbits after the equilibrium points of a differential system. Their study is interesting because the motion in their neighborhood can be determined by their type of stability. For a given Hamiltonian system usually it is very difficult to study analytically the existence of its periodic orbits and their kind of stability.

In order to apply the averaging theory to differential system (2), for studying analytically its periodic solutions, we introduce a small parameter $\varepsilon$ by scaling the variables $\left(X, Y, Z, p_{X}, p_{Y}, p_{Z}\right)=$ $\left(\sqrt{\varepsilon} x, \sqrt{\varepsilon} y, \sqrt{\varepsilon} z, \sqrt{\varepsilon} p_{x}, \sqrt{\varepsilon} p_{y}, \sqrt{\varepsilon} p_{z}\right)$. This change of coordinates is canonical, and developing in these new variables the Hamiltonian (1) in Taylor series around $\varepsilon=0$ we get

$$
\begin{aligned}
\mathcal{H}= & \frac{1}{2}\left(p_{x}^{2}+p_{y}^{2}+p_{z}^{2}\right) \varepsilon+\frac{1}{2(a+b)^{3}}\left(x^{2}+y^{2}+\frac{(a+b)}{b} z^{2}\right)- \\
& \frac{1}{8(a+b)^{5}}\left[3\left(x^{2}+y^{2}+\frac{(a+b)}{b} z^{2}\right)^{2}+\frac{a(a+b)^{2}}{b^{3}} z^{4}\right] \varepsilon^{2}+O\left(\varepsilon^{3}\right),
\end{aligned}
$$

and the Hamiltonian system (2) becomes

$$
\begin{aligned}
\dot{x} & =p_{x}, \\
\dot{y} & =p_{y}, \\
\dot{z} & =p_{z}, \\
\dot{p}_{x} & =-\frac{x}{(a+b)^{3}}+\frac{3}{2(a+b)^{5}} x\left(x^{2}+y^{2}+\frac{(a+b)}{b} z^{2}\right) \varepsilon+O\left(\varepsilon^{2}\right), \\
\dot{p}_{y} & =-\frac{y}{(a+b)^{3}}+\frac{3}{2(a+b)^{5}} y\left(x^{2}+y^{2}+\frac{(a+b)}{b} z^{2}\right) \varepsilon+O\left(\varepsilon^{2}\right), \\
\dot{p}_{z} & =-\frac{z}{b(a+b)^{2}}+z \frac{3 b^{2}\left(x^{2}+y^{2}\right)+(a+b)(a+3 b) z^{2}}{2 b^{3}(a+b)^{4}} \varepsilon+O\left(\varepsilon^{2}\right) .
\end{aligned}
$$

We proceed to study of the existence of periodic solutions of system (4). First we analyze the solutions of system (4) with $\varepsilon=0$, that is, the unperturbed Hamiltonian system

$$
\begin{aligned}
& \dot{x}=p_{X}, \quad \dot{p}_{X}=-\frac{x}{(a+b)^{3}}, \\
& \dot{y}=p_{Y}, \quad \dot{p}_{Y}=-\frac{y}{(a+b)^{3}}, \\
& \dot{z}=p_{Z}, \quad \dot{p}_{Z}=-\frac{z}{b(a+b)^{2}},
\end{aligned}
$$


The solutions of this unperturbed system, which is a sum of three harmonic oscillators, with arbitrary initial conditions $x(0)=x_{0}, y(0)=y_{0}, z(0)=z_{0}, p_{x}(0)=p_{x_{0}}, p_{y}(0)=p_{y_{0}}, p_{z}(0)=$ $p_{z_{0}}$ are

$$
\begin{aligned}
x(t) & =x_{0} \cos \omega_{1} t+\frac{1}{\omega_{1}} p_{x_{0}} \sin \omega_{1} t \\
p_{x}(t) & =p_{x_{0}} \cos \omega_{1} t-\omega_{1} x_{0} \sin \omega_{1} t \\
y(t) & =y_{0} \cos \omega_{1} t+\frac{1}{\omega_{1}} p_{y_{0}} \sin \omega_{1} t \\
p_{y}(t) & =p_{y_{0}} \cos \omega_{1} t-\omega_{1} y_{0} \sin \omega_{1} t \\
z(t) & =z_{0} \cos \omega_{2} t+\frac{1}{\omega_{2}} p_{z_{0}} \sin \omega_{2} t \\
p_{z}(t) & =p_{z_{0}} \cos \omega_{2} t-\omega_{2} z_{0} \sin \omega_{2} t
\end{aligned}
$$

where $\omega_{1}=(a+b)^{-3 / 2}$ and $\omega_{2}=b^{-1 / 2}(a+b)^{-1}$. Since $\frac{\omega_{2}}{\omega_{1}}=\sqrt{\frac{a+b}{b}}$, then for the unperturbed system the following statement hold:

(i) If $\sqrt{\frac{a+b}{b}}$ is rational, then the set of all periodic solutions of system (5) has dimension 6 contained in $\mathbb{R}^{6}$.

(ii) If $\sqrt{\frac{a+b}{b}}$ is irrational, then there are two sets of periodic solutions of system (5) one of dimension 4 and the other of dimension 2. The first family is contained in the subspace $\left(x, y, 0, p_{x}, p_{y}, 0\right)$ and the second is contained in $\left(0,0, z, 0,0, p_{z}\right)$.

Now we are in position to state our main results.

The second result is generated using the periodic solutions on the subspace $\left(x, y, 0, p_{x}, p_{y}, 0\right)$ and is the following.

Theorem 1. If $\sqrt{\frac{a+b}{b}}$ is irrational, then for $\varepsilon>0$ sufficiently small at every level $\mathcal{H}=$ $h>0$ the perturbed Hamiltonian systems (4) has at least one periodic solution $\gamma(t, \varepsilon)=$ $\left(x(t, \varepsilon), y(t, \varepsilon), z(t, \varepsilon), p_{x}(t, \varepsilon), p_{y}(t, \varepsilon), p_{z}(t, \varepsilon)\right)$ with period $T=2 \pi(a+b)^{3 / 2}$ such that $\gamma(0, \varepsilon) \rightarrow$ $\left(\sqrt{2(a+b)^{3} h}, 0,0,0,0,0\right)$ as $\varepsilon \rightarrow 0$.

Details of the proof of Theorem 1 will be given in section 2 .

Theorem 2. If $\sqrt{\frac{a+b}{b}}$ is irrational, then for $\varepsilon>0$ sufficiently small at every level $\mathcal{H}=$ $h>0$ the perturbed Hamiltonian systems (4) has at least one periodic solution $\gamma(t, \varepsilon)=$ $\left(x(t, \varepsilon), y(t, \varepsilon), z(t, \varepsilon), p_{x}(t, \varepsilon), p_{y}(t, \varepsilon), p_{z}(t, \varepsilon)\right)$ with period $T=2 \pi(a+b) \sqrt{b}$ such that $\gamma(0, \varepsilon) \rightarrow$ $(0,0,(a+b) \sqrt{2 b h}, 0,0,0)$ as $\varepsilon \rightarrow 0$.

The proof of Theorem 2 will be given in section 3 .

Both theorems are proved using the averaging theory described in the appendix. Unfortunately this averaging theory does not provide any information about the periodic orbits (i) of the unperturbed system (5) which can be continued to system (4).

\section{Proof of Theorem 1}

We consider the unperturbed periodic solutions defined in (6) with $z=p_{z_{0}}=0$, that is, we take the periodic solution with four parameters 


$$
\begin{aligned}
\gamma_{0}(t)= & \left(x_{0} \cos \left(\omega_{1} t\right)+\frac{1}{\omega_{1}} p_{x_{0}} \sin \left(\omega_{1} t\right), y_{0} \cos \left(\omega_{1} t\right)+\frac{1}{\omega_{1}} p_{y_{0}} \sin \left(\omega_{1} t\right), 0\right. \\
& \left.p_{x_{0}} \cos \left(\omega_{1} t\right)-\omega_{1} x_{0} \sin \left(\omega_{1} t\right), p_{y_{0}} \cos \left(\omega_{1} t\right)-\omega_{1} y_{0} \sin \left(\omega_{1} t\right), 0\right),
\end{aligned}
$$

with period $T=2 \pi / \omega_{1}=2 \pi(a+b)^{3 / 2}$ which are in the subspace $\left(x, y, 0, p_{x}, p_{y}, 0\right)$. Note that these periodic solutions are not in resonance with the other periodic solutions of system (5) living in the subspace $\left(0,0, z, 0,0, p_{z}\right)$ due to the fact that $\omega_{1} / \omega_{2}$ is not a rational number by assumptions. It is clear that the energy level of this family of periodic solutions with initial conditions $\left(x_{0}, y_{0}, 0, p_{x_{0}}, p_{y_{0}}, 0\right)$ and $\varepsilon=0$ is

$$
h=\frac{1}{2}\left(\omega_{1}^{2}\left(x_{0}^{2}+y_{0}^{2}\right)+p_{x_{0}}^{2}+p_{y_{0}}^{2}\right) .
$$

We are going to apply Theorem 3 of the appendix at every energy level $\mathcal{H}=h>0$, this will allow to eliminate one variable, in our case it will be $p_{x}$, and to reduce the study in a space of dimension five. Using the expression of $\mathcal{H}$ given in (3) we get

$$
\begin{aligned}
& p_{x}=\sqrt{2 h-\frac{b\left((a+b)^{3}\left(p_{y}^{2}+p_{z}^{2}\right)+x^{2}+y^{2}\right)+z^{2}(a+b)}{b(a+b)^{3}}}+ \\
& \frac{6 b^{2} z^{2}(a+b)\left(x^{2}+y^{2}\right)+z^{4}(a+b)^{2}(a+3 b)+3 b^{3}\left(x^{2}+y^{2}\right)^{2}}{8 b^{3}(a+b)^{5} \sqrt{\frac{b\left((a+b)^{3}\left(2 h-p_{y}^{2}-p_{z}^{2}\right)-x^{2}-y^{2}\right)-z^{2}(a+b)}{b(a+b)^{3}}}} \varepsilon+O\left(\varepsilon^{2}\right) .
\end{aligned}
$$

We consider the following order of the variables $\left(x, y, p_{y}, z, p_{z}\right)$, it is verified that the equations of motion (4) on the energy level $\mathcal{H}=h>0$ are given by

$$
\begin{aligned}
& \dot{x}=\sqrt{2 h-\frac{b\left((a+b)^{3}\left(p_{y}^{2}+p_{z}^{2}\right)+x^{2}+y^{2}\right)+z^{2}(a+b)}{b(a+b)^{3}}}+ \\
& \frac{6 b^{2}(a+b) z^{2}\left(x^{2}+y^{2}\right)+(a+b)^{2}(a+3 b) z^{4}+3 b^{3}\left(x^{2}+y^{2}\right)^{2}}{8 b^{3}(a+b)^{5} \sqrt{\frac{b\left((a+b)^{3}\left(2 h-p_{y}^{2}-p_{z}^{2}\right)-x^{2}-y^{2}\right)-z^{2}(a+b)}{b(a+b)^{3}}}} \varepsilon+O\left(\varepsilon^{2}\right), \\
& \dot{y}=p_{y}, \\
& \dot{p}_{y}=-\frac{y}{(a+b)^{3}}+\frac{3 y\left(\frac{z^{2}(a+b)}{b}+x^{2}+y^{2}\right)}{2(a+b)^{5}} \varepsilon+O\left(\varepsilon^{2}\right), \\
& \dot{z}=p_{z} \text {, } \\
& \dot{p}_{z}=-\frac{z}{b(a+b)^{2}}+\frac{(a+b)(a+3 b) z^{3}+3 b^{2} z\left(x^{2}+y^{2}\right)}{2 b^{3}(a+b)^{4}} \varepsilon+O\left(\varepsilon^{2}\right) .
\end{aligned}
$$

This differential system has the normal form of system (8), so it is ready for applying to it the averaging theory described in the appendix, i.e. the function $F_{0}\left(x, y, p_{y}, z, p_{z}\right)$ is

$$
\begin{aligned}
& \left(\sqrt{2 h-\frac{b\left((a+b)^{3}\left(p_{y}^{2}+p_{z}^{2}\right)+x^{2}+y^{2}\right)+z^{2}(a+b)}{b(a+b)^{3}}}, p_{y},-\frac{y}{(a+b)^{3}}+\right. \\
& \left.\frac{3 y\left(\frac{z^{2}(a+b)}{b}+x^{2}+y^{2}\right)}{2(a+b)^{5}}, p_{z},-\frac{z}{b(a+b)^{2}}+\frac{(a+b)(a+3 b) z^{3}+3 b^{2} z\left(x^{2}+y^{2}\right)}{2 b^{3}(a+b)^{4}}\right),
\end{aligned}
$$


and the function $F_{1}\left(x, y, p_{y}, z, p_{z}\right)$ is

$$
\begin{aligned}
& \left(\frac{6 b^{2}(a+b) z^{2}\left(x^{2}+y^{2}\right)+(a+b)^{2}(a+3 b) z^{4}+3 b^{3}\left(x^{2}+y^{2}\right)^{2}}{8 b^{3}(a+b)^{5} \sqrt{\frac{b\left((a+b)^{3}\left(2 h-p_{y}^{2}-p_{z}^{2}\right)-x^{2}-y^{2}\right)-z^{2}(a+b)}{b(a+b)^{3}}}, 0,}\right. \\
& \left.\frac{3 y\left(\frac{z^{2}(a+b)}{b}+x^{2}+y^{2}\right)}{2(a+b)^{5}}, 0, \frac{(a+b)(a+3 b) z^{3}+3 b^{2} z\left(x^{2}+y^{2}\right)}{2 b^{3}(a+b)^{4}}\right) .
\end{aligned}
$$

In what follows we will use the notation of Theorem 3 given in the appendix, that is, $k=3$, $n=5, \xi\left(x, y, p_{y}, z, p_{z}\right)=\left(x, y, p_{y}\right), \alpha=\left(x_{0}, y_{0}, p_{y_{0}}\right), \beta(\alpha)=(0,0)$. So the initial condition of the unperturbed periodic orbits of system (5) in the energy level $\mathcal{H}=h>0$ is $x_{\alpha}=$ $\left(x_{0}, y_{0}, p_{y_{0}}, 0,0\right)$. For each $x_{\alpha}$ the solution $x\left(t, x_{\alpha}\right)$ equal to

$$
\left(x_{0} \cos \left(\omega_{1} t\right)+\frac{1}{\omega_{1}} p_{x_{0}} \sin \left(\omega_{1} t\right), y_{0} \cos \left(\omega_{1} t\right)+\frac{1}{\omega_{1}} p_{y_{0}} \sin \left(\omega_{1} t\right), p_{y_{0}} \cos \left(\omega_{1} t\right)-\omega_{1} y_{0} \sin \left(\omega_{1} t\right), 0,0\right)
$$

is $T=\frac{2 \pi}{\omega_{1}}=2 \pi(a+b)^{3 / 2}$-periodic. Let $M_{x_{\alpha}}(t)$ be the fundamental matrix satisfying $M_{x_{\alpha}}(0)$ is the identity matrix, solution of the variational equation (10) along the periodic solution $x\left(t, x_{\alpha}\right)$. Then, $M_{x_{\alpha}}(t)$ is

$$
\left(\begin{array}{ccccc}
\cos \left(\omega_{1} t\right)-\omega_{1} \frac{x_{0}}{p_{x_{0}}} \sin \left(\omega_{1} t\right) & -\omega_{1} \frac{y_{0}}{p_{x_{0}}} \sin \left(\omega_{1} t\right) & -\frac{1}{\omega_{1}} \frac{p_{y_{0}}}{p_{x_{0}}} \sin \left(\omega_{1} t\right) & 0 & 0 \\
0 & \cos \left(\omega_{1} t\right) & \frac{1}{\omega_{1}} \sin \left(\omega_{1} t\right) & 0 & 0 \\
0 & -\omega_{1} \sin \left(\omega_{1} t\right) & \cos \left(\omega_{1} t\right) & 0 & 0 \\
0 & 0 & 0 & \cos \left(\omega_{2} t\right) & \frac{1}{\omega_{2}} \sin \left(\omega_{2} t\right) \\
0 & 0 & 0 & -\omega_{2} \sin \left(\omega_{1} t\right) & \cos \left(\omega_{1} t\right)
\end{array}\right)
$$

In order to apply Theorem 3 of the appendix we need to compute the matrix $M_{x_{\alpha}}^{-1}(0)-$ $M_{x_{\alpha}}^{-1}(T)$, which is

$$
\left(\begin{array}{ccccc}
0 & 0 & 0 & 0 & 0 \\
0 & 0 & 0 & 0 & 0 \\
0 & 0 & 0 & 0 & 0 \\
0 & 0 & 0 & 2 \sin ^{2}\left(\frac{\sqrt{a+b}}{\sqrt{b}} \pi\right) & \sqrt{b}(a+b) \sin \left(\frac{2 \sqrt{a+b}}{\sqrt{b}} \pi\right) \\
& & & \sin \left(\frac{2 \sqrt{a+b}}{\sqrt{b}} \pi\right) & 2 \sin ^{2}\left(\frac{\sqrt{a+b}}{\sqrt{b}} \pi\right)
\end{array}\right)
$$

It is clear that the upper right corner the $3 \times 2$ is the zero matrix, and in the lower right corner we have a $2 \times 2$ matrix $\Delta_{\alpha}$ with $\operatorname{det} \Delta_{\alpha}=4 \sin ^{2}\left(\frac{\pi \sqrt{a+b}}{\sqrt{b}}\right) \neq 0$, because $\frac{\sqrt{b}}{\sqrt{a+b}}$ is an irrational number. Now we compute the averaged function defined in (11), i.e.

$$
\begin{aligned}
\mathcal{F}\left(x_{0}, y_{0}, p_{y_{0}}\right) & =\xi\left(\int_{0}^{2 \pi(a+b)^{3 / 2}} M_{x_{\alpha}}^{-1}(t) F_{1}\left(t, x\left(t, x_{\alpha}\right)\right) d t\right) \\
& =\left(f_{11}, f_{12}, f_{13}\right),
\end{aligned}
$$


and we get

$$
\begin{aligned}
& f_{11}=\frac{3 \pi}{8 \sqrt{a+b}}\left[\left(-3 p_{x_{0}}(a+b)^{3}\left(p_{x_{0}}^{2}+p_{y_{0}}^{2}\right)-3 p_{x_{0}} x_{0}^{2}-p_{x_{0}} y_{0}^{2}-2 p_{y_{0}} x_{0} y_{0}\right)\right] \\
& f_{12}=-\frac{3 \pi}{8 \sqrt{a+b}}\left[\left(p_{y_{0}}\left(3(a+b)^{3}\left(p_{x_{0}}^{2}+p_{y_{0}}^{2}\right)+x_{0}^{2}\right)+2 p_{x_{0}} x_{0} y_{0}+3 p_{y_{0}} y_{0}^{2}\right)\right] \\
& f_{13}=\frac{3 \pi}{8(a+b)^{7 / 2}}\left[\left(y_{0}\left((a+b)^{3}\left(p_{x_{0}}^{2}+3 p_{y_{0}}^{2}\right)+3 x_{0}^{2}\right)+2(a+b)^{3} p_{x_{0}} p_{y_{0}} x_{0}+3 y_{0}^{3}\right)\right]
\end{aligned}
$$

where $p_{x_{0}}= \pm \sqrt{2 h-p_{y_{0}}^{2}-\frac{x_{0}^{2}+y_{0}^{2}}{(a+b)^{3}}}$. Solving $\mathcal{F}\left(x_{0}, y_{0}, p_{y_{0}}\right)=0$, we obtain the two real solutions $x_{0}^{*}= \pm \sqrt{2 h(a+b)^{3}}, y_{0}=p_{y_{0}}=0$. It is verified that the differential $D \mathcal{F}\left(x_{0}, y_{0}, p_{y_{0}}\right)=$ $\left(d f_{i j}\right)$, where

$$
\begin{aligned}
& d f_{11}=-\frac{3 \pi}{4 \sqrt{a+b}}\left(p_{y_{0}} y_{0}-\frac{x_{0}\left(3(a+b)^{3} h-y_{0}^{2}\right)}{(a+b)^{3} \sqrt{-\frac{-\left(2 h-p_{y_{0}}^{2}\right)(a+b)^{3}+x_{0}^{2}+y_{0}^{2}}{(a+b)^{3}}}}\right), \\
& d f_{12}=-\frac{3 \pi}{4 \sqrt{a+b}}\left(p_{y_{0}} x_{0}-2 y_{0} \sqrt{-\frac{-\left(2 h-p_{y_{0}}^{2}\right)(a+b)^{3}+x_{0}^{2}+y_{0}^{2}}{(a+b)^{3}}}\right. \\
& \left.-\frac{y_{0}\left(3(a+b)^{3} h-y_{0}^{2}\right)}{(a+b)^{3} \sqrt{-\frac{-\left(2 h-p_{y_{0}}^{2}\right)(a+b)^{3}+x_{0}^{2}+y_{0}^{2}}{(a+b)^{3}}}}\right) \text {, } \\
& d f_{13}=-\frac{3 \pi}{4 \sqrt{a+b}}\left(x_{0} y_{0}-\frac{p_{y_{0}}\left(3(a+b)^{3} h-y_{0}^{2}\right)}{\sqrt{-\frac{-\left(2 h-p_{y_{0}}^{2}\right)(a+b)^{3}+x_{0}^{2}+y_{0}^{2}}{(a+b)^{3}}}}\right) \text {, } \\
& d f_{21}=-\frac{3 \pi}{4 \sqrt{a+b}}\left(-\frac{y_{0} x_{0}^{2}}{(a+b)^{3} \sqrt{-p_{y_{0}}^{2}+2 h-\frac{x_{0}^{2}+y_{0}^{2}}{(a+b)^{3}}}}-2 p_{y_{0}} x_{0}+y_{0} \sqrt{2 h-\frac{x_{0}^{2}+y_{0}^{2}}{(a+b)^{3}}-p_{y_{0}}^{2}}\right), \\
& d f_{22}=-\frac{3 \pi x_{0}\left(2 h-\frac{x_{0}^{2}+y_{0}^{2}}{(a+b)^{3}}-p_{y_{0}}^{2}\right)}{4 \sqrt{a+b} \sqrt{2 h-\frac{x_{0}^{2}+y_{0}^{2}}{(a+b)^{3}}-p_{y_{0}}^{2}}}, \\
& d f_{23}=-\frac{3 \pi}{4 \sqrt{a+b}}\left(3 h a^{3}+9 b h a^{2}+9 b^{2} h a-x_{0}^{2}+3 b^{3} h-\frac{p_{y_{0}} x_{0} y_{0}}{\sqrt{2 h-\frac{x_{0}^{2}+y_{0}^{2}}{(a+b)^{3}}-p_{y_{0}}^{2}}}\right) \text {, }
\end{aligned}
$$




$$
\begin{aligned}
& d f_{31}=\frac{3 \pi}{8(a+b)^{7 / 2}}\left(2 p_{y_{0}} \sqrt{2 h-\frac{x_{0}^{2}+y_{0}^{2}}{(a+b)^{3}}-p_{y_{0}}^{2}}(a+b)^{3}+4 x_{0} y_{0}-\frac{2 p_{y_{0}} x_{0}^{2}}{\sqrt{2 h-\frac{x_{0}^{2}+y_{0}^{2}}{(a+b)^{3}}-p_{y_{0}}^{2}}}\right), \\
& d f_{32}=\frac{3 \pi}{4(a+b)^{7 / 2}}\left(\begin{array}{l}
\left(p_{y_{0}}^{2}+h\right)(a+b)^{3}+x_{0}^{2}+3 y_{0}^{2}-\frac{p_{y_{0}} x_{0} y_{0}}{\sqrt{2 h-\frac{x_{0}^{2}+y_{0}^{2}}{(a+b)^{3}}-p_{y_{0}}^{2}}}, \\
d f_{33}=\frac{3 \pi}{4 \sqrt{a+b}}\left(-\frac{x_{0} p_{y_{0}}^{2}}{\sqrt{2 h-\frac{x_{0}^{2}+y_{0}^{2}}{(a+b)^{3}}-p_{y_{0}}^{2}}}+2 y_{0} p_{y_{0}}+x_{0} \sqrt{2 h-\frac{x_{0}^{2}+y_{0}^{2}}{(a+b)^{3}}-p_{y_{0}}^{2}}\right) .
\end{array}\right)
\end{aligned}
$$

It is verified that the determinant (12) is not zero, i.e.

$$
\operatorname{det}\left(\mathcal{F}\left(x_{0}, 0,0\right)\right)=\frac{243 \pi^{3} h^{3} x_{0}(a+b)^{3 / 2}}{64 \sqrt{2 h-\frac{x_{0}^{2}}{(a+b)^{3}}}} .
$$

Thus the determinant evaluated at $x_{0}^{*}= \pm \sqrt{2 h(a+b)^{3}}$, which is different from zero, in fact, is $\infty$. Since both solutions generate the same periodic orbit, we conclude the proof.

\section{Proof of Theorem 2}

We consider the unperturbed periodic solutions defined in (6) with $x_{0}=y_{0}=p_{x_{0}}=p_{y_{0}}=0$, that is, we take the periodic solution with two parameters

$$
\gamma_{0}(t)=\left(0,0, z_{0} \cos \omega_{2} t+\frac{1}{\omega_{2}} p_{z_{0}} \sin \omega_{2} t, 0,0, p_{z_{0}} \cos \omega_{2} t-\omega_{2} z_{0} \sin \omega_{2} t\right)
$$

and with period $T=\frac{2 \pi}{\omega_{2}}$ which is in the plane $\left(0,0, z, 0,0, p_{z}\right)$. Note that these periodic solutions are not in resonance with the other periodic solutions of system (5) living in the subspace $\left(x, y, 0, p_{x}, p_{y}, 0\right)$ due to the fact that $\omega_{1} / \omega_{2}$ is not a rational number. It is clear that the energy level of this family of periodic solutions with $\varepsilon=0$ is

$$
h=\frac{1}{2}\left(\omega_{2}^{2} z_{0}^{2}+p_{z_{0}}^{2}\right)
$$

Again we apply Theorem 3 of appendix at every energy level $\mathcal{H}=h>0$, this allows to eliminate one variable, now the variable $p_{z}$, and we reduce the space of motion to dimension five. Using the expression of $\mathcal{H}$ in (3) we get

$$
\begin{aligned}
p_{z}= & \sqrt{2 h-\frac{b\left((a+b)^{3}\left(p_{x}^{2}+p_{y}^{2}\right)+x^{2}+y^{2}\right)+z^{2}(a+b)}{b(a+b)^{3}}}+ \\
& \frac{6 b^{2} z^{2}(a+b)\left(x^{2}+y^{2}\right)+z^{4}(a+b)^{2}(a+3 b)+3 b^{3}\left(x^{2}+y^{2}\right)^{2}}{8 b^{5 / 2}(a+b)^{7 / 2} \sqrt{b\left((a+b)^{3}\left(2 h-\mathrm{px}^{2}-\mathrm{py}^{2}\right)-x^{2}-y^{2}\right)-z^{2}(a+b)}} \varepsilon+O\left(\varepsilon^{2}\right) .
\end{aligned}
$$


We will consider the following order of the variables $\left(z, x, p_{x}, y, p_{y}\right)$, it is verified that the equations of motion (4) on the energy level $\mathcal{H}=h$ are given by

(7)

$$
\begin{aligned}
\dot{z}= & \sqrt{2 h-\frac{b\left((a+b)^{3}\left(p_{x}^{2}+p_{y}^{2}\right)+x^{2}+y^{2}\right)+z^{2}(a+b)}{b(a+b)^{3}}}+ \\
& \frac{6 b^{2} z^{2}(a+b)\left(x^{2}+y^{2}\right)+z^{4}(a+b)^{2}(a+3 b)+3 b^{3}\left(x^{2}+y^{2}\right)^{2}}{8 b^{5 / 2}(a+b)^{7 / 2} \sqrt{b\left((a+b)^{3}\left(2 h-p_{x}^{2}-p_{y}^{2}\right)-x^{2}-y^{2}\right)-z^{2}(a+b)}} \varepsilon+O\left(\varepsilon^{2}\right), \\
\dot{x}= & p_{x}, \\
\dot{p}_{x}= & -\frac{x}{(a+b)^{3}}+\frac{3}{2(a+b)^{5}} x\left(x^{2}+y^{2}+\frac{(a+b)}{b} z^{2}\right) \varepsilon+O\left(\varepsilon^{2}\right), \\
\dot{y}= & p_{y}, \\
\dot{p}_{y}= & -\frac{y}{(a+b)^{3}}+\frac{3}{2(a+b)^{5}} y\left(x^{2}+y^{2}+\frac{(a+b)}{b} z^{2}\right) \varepsilon+O\left(\varepsilon^{2}\right) .
\end{aligned}
$$

This differential system has the normal form of system (8) for applying to it the averaging theory of the appendix, where

$$
\begin{aligned}
F_{0}\left(z, x, p_{x}, y, p_{y}\right)= & \left(\sqrt{2 h-\frac{b\left((a+b)^{3}\left(p_{x}^{2}+p_{y}^{2}\right)+x^{2}+y^{2}\right)+z^{2}(a+b)}{b(a+b)^{3}}},\right. \\
& \left.p_{x},-\frac{x}{(a+b)^{3}}, p_{y},-\frac{y}{(a+b)^{3}}\right)
\end{aligned}
$$

and $F_{1}\left(z, x, p_{x}, y, p_{y}\right)$ is

$$
\begin{aligned}
& \left(\frac{6 b^{2} z^{2}(a+b)\left(x^{2}+y^{2}\right)+z^{4}(a+b)^{2}(a+3 b)+3 b^{3}\left(x^{2}+y^{2}\right)^{2}}{8 b^{5 / 2}(a+b)^{7 / 2} \sqrt{b\left((a+b)^{3}\left(2 h-p_{x}^{2}-p_{y}^{2}\right)-x^{2}-y^{2}\right)-z^{2}(a+b)}}, 0,\right. \\
& \left.\frac{3}{2(a+b)^{5}} x\left(x^{2}+y^{2}+\frac{(a+b)}{b} z^{2}\right), 0, \frac{3}{2(a+b)^{5}} y\left(x^{2}+y^{2}+\frac{(a+b)}{b} z^{2}\right)\right) .
\end{aligned}
$$

In what follows we will use the notation of Theorem 3 given in the appendix, that is, $k=1, n=$ $5, \xi\left(z, x, p_{x}, y, p_{y}\right)=z, \alpha=z_{0}, \beta(\alpha)=(0,0,0,0)$, and $z_{\alpha}=\left(z_{0}, 0,0,0,0\right)$ is the initial condition of a periodic orbit of the unperturbed system (9) corresponding to system (7). For each $z_{\alpha}$ the solution $x\left(t, z_{\alpha}\right)=\left(z_{0} \cos \omega_{2} t+\frac{1}{\omega_{2}} p_{z_{0}} \sin \omega_{2} t, 0,0,0,0\right)$ has period $T=\frac{2 \pi}{\omega_{2}}=2 \pi(a+b) \sqrt{b}$. Let $M_{x_{\alpha}}(t)$ be the fundamental matrix of the variational equation (10) along the periodic solutions $x\left(t, z_{\alpha}\right)$ such that $M_{x_{\alpha}}(0)$ is the identity matrix, . Then $M_{x_{z_{0}}}(t)$ is

$$
\left(\begin{array}{ccccc}
\cos \left(\omega_{2} t\right)-\omega_{2} \frac{z_{0}}{p_{z_{0}}} \sin \left(\omega_{2} t\right) & 0 & 0 & 0 & 0 \\
0 & \cos \left(\omega_{1} t\right) & \frac{1}{\omega_{1}} \sin \left(\omega_{1} t\right) & 0 & 0 \\
0 & -\omega_{1} \sin \left(\omega_{1} t\right) & \cos \left(\omega_{1} t\right) & 0 & 0 \\
0 & 0 & 0 & \cos \left(\omega_{1} t\right) & \frac{1}{\omega_{1}} \sin \left(\omega_{1} t\right) \\
0 & 0 & 0 & -\omega_{1} \sin \left(\omega_{1} t\right) & \cos \left(\omega_{1} t\right)
\end{array}\right) .
$$


Next in order to apply Theorem 3 of the appendix we compute the matrix $M_{x_{z_{0}}}^{-1}(0)-M_{x_{z_{0}}}^{-1}(T)$, and we obtain

$$
\left(\begin{array}{ccccc}
0 & 0 & 0 & 0 \\
0 & 2 \sin ^{2}\left(\frac{\sqrt{b} \pi}{\sqrt{a+b}}\right) & (a+b)^{3 / 2} \sin \left(\frac{2 \sqrt{b} \pi}{\sqrt{a+b}}\right) & 0 & 0 \\
& \begin{array}{c}
\sin \left(\frac{2 \sqrt{b} \pi}{\sqrt{a+b}}\right) \\
0
\end{array}-\frac{2 \sin ^{2}\left(\frac{\sqrt{b} \pi}{\sqrt{a+b}}\right)}{(a+b)^{3 / 2}} & 0 & 0 \\
0 & 0 & 0 & 2 \sin ^{2}\left(\frac{\sqrt{b} \pi}{\sqrt{a+b}}\right) & (a+b)^{3 / 2} \sin \left(\frac{2 \sqrt{b} \pi}{\sqrt{a+b}}\right) \\
0 & 0 & 0 & -\frac{\sin \left(\frac{2 \sqrt{b} \pi}{\sqrt{a+b}}\right)}{(a+b)^{3 / 2}} & 2 \sin ^{2}\left(\frac{\sqrt{b} \pi}{\sqrt{a+b}}\right)
\end{array}\right) .
$$

It is clear that the upper right corner the $1 \times 4$ is the zero matrix, and in the lower right corner we have a $4 \times 4$ matrix $\Delta_{z_{0}}$ with $\operatorname{det} \Delta_{\alpha}=16 \sin ^{4}\left(\frac{\pi \sqrt{b}}{\sqrt{a+b}}\right) \neq 0$, because $\frac{\sqrt{b}}{\sqrt{a+b}}$ is an irrational number. Then we have

$$
\begin{aligned}
\mathcal{F}\left(z_{0}\right) & =\xi\left(\int_{0}^{2 \pi(a+b) \sqrt{b}} M_{x_{z_{0}}}^{-1}(t) F_{1}\left(t, x\left(t, x_{z_{0}}\right)\right) d t\right) \\
& =-\frac{3 \pi h(a+b)^{2}(a+3 b) \sqrt{2 h-\frac{z_{0}^{2}}{b(a+b)^{2}}}}{4 \sqrt{b}} .
\end{aligned}
$$

Solving $\mathcal{F}\left(z_{0}\right)=0$ we obtain the two solutions $z_{0}=z_{0}^{*}= \pm(a+b) \sqrt{2 b h}$, for which we have $p_{z_{0}}=0$. It is verified that the derivative $\mathcal{F}^{\prime}\left(z_{0}\right)=\frac{3 \pi h z_{0}(a+3 b)}{4 b^{3 / 2} \sqrt{2 h-\frac{z_{0}^{2}}{b(a+b)^{2}}}}$, which is different from zero at $z_{0}=z_{0}^{*}$, in fact, it is $\infty$. Since both values of $z_{0}$ generate the same periodic orbit, we conclude the proof.

\section{Appendix: Averaging theOry of FIRST AND SECOND ORDER}

In this section we recall the averaging theory of first order to find periodic orbits, for more information on the general averaging theory see the book [14].

Consider the differential system

$$
\dot{\mathbf{x}}=F_{0}(t, \mathbf{x})+\varepsilon F_{1}(t, \mathbf{x})+\varepsilon^{2} F_{2}(t, \mathbf{x}, \varepsilon)
$$

with $\varepsilon>0$ sufficiently small, $\mathbf{x} \in \Omega$, where $\Omega$ is an open subset of $\mathbb{R}^{n}$, and $t \geq 0$. Moreover, we assume that both $F_{0}, F_{1}$ and $F_{2}$ are $\mathcal{C}^{2}$ functions and $T$-periodic in the first variable.

Let $\mathbf{x}(t, \mathbf{z})$ be the solution of the unperturbed system

$$
\dot{\mathbf{x}}=F_{0}(t, \mathbf{x}),
$$

such that $\mathbf{x}(0, \mathbf{z})=\mathbf{z}$.

We write the linearization of the unperturbed system along the periodic solution $\mathbf{x}(t, \mathbf{z})$ as

$$
\dot{\mathbf{y}}(t)=D_{\mathbf{x}} F_{0}(t, \mathbf{x}(t, \mathbf{z})) \mathbf{y} .
$$


Now we denote by $M_{\mathbf{z}}(t)$ some fundamental matrix of the linear differential system (10), and by $\xi: \mathbb{R}^{k} \times \mathbb{R}^{n-k} \longrightarrow \mathbb{R}^{k}$, the projection of $\mathbb{R}^{n}$ onto its first $k$ coordinates; i.e., $\xi\left(x_{1}, \ldots, x_{n}\right)=$ $\left(x_{1}, \ldots, x_{k}\right)$.

Theorem 3 (First Order Averaging Theorem). Let $V \subset \mathbb{R}^{k}$ be open and bounded, and let $\beta_{0}: C L(V) \longrightarrow \mathbb{R}^{n-k}$ be a $\mathcal{C}^{2}$ function. We assume that

(i) $\mathcal{Z}=\left\{\mathbf{z}_{\alpha}=\left(\alpha, \beta_{0}(\alpha)\right), \alpha \in C l(V)\right\} \subset \Omega$ and that for each $\mathbf{z}_{\alpha} \in \mathcal{Z}$ the solution $\mathbf{x}\left(t, \mathbf{z}_{\alpha}\right)$ of (9) is T-periodic;

(ii) for each $\mathbf{z}_{\alpha} \in \mathcal{Z}$ there is a fundamental matrix $M_{\mathbf{z}_{\alpha}}(t)$ of (10) such that the matrix $M_{\mathbf{z}_{\alpha}}^{-1}(0)-M_{\mathbf{z}_{\alpha}}^{-1}(T)$ has in the upper right corner the $k \times(n-k)$ zero matrix, and in the lower right corner $a(n-k) \times(n-k)$ matrix $\Delta_{\alpha}$ with det $\Delta_{\alpha} \neq 0$.

We consider the function $\mathcal{F}(\alpha): C l(V) \rightarrow \mathbb{R}^{k}$

$$
\mathcal{F}(\alpha)=\xi\left(\int_{0}^{T} M_{\mathbf{z}_{\alpha}}^{-1}(t) F_{1}\left(t, \mathbf{x}\left(t, \mathbf{z}_{\alpha}\right)\right) d t\right) .
$$

If there exists $a \in V$ with $\mathcal{F}(a)=0$ and

$$
\operatorname{det}((d \mathcal{F} / d \alpha(a)) \neq 0,
$$

then there is a T-periodic solution $\mathbf{x}(t, \varepsilon)$ of system (8) such that $\mathbf{x}(0, \varepsilon) \rightarrow$ a as $\varepsilon \rightarrow 0$.

For an easy proof of Theorem 3 see [2]. In fact the result of Theorem 3 is a classical result due to Malkin [9] and Roseau [13].

\section{ACKNOWLEDGMENTS}

The first author is partially supported by the MINECO grants MTM2013-40998-P and MTM2016-77278-P (FEDER), the AGAUR grant 2014 SGR568, and the projecte MDM-20140445 (BGSMath).

The second author is partially supported by Fondecyt project 1130644 .

\section{REFERENCES}

[1] Binney, J.: More dynamical models of our Galaxy. Mon. Not. R. Astron. Soc. 426, 1328-1337 (2012)

[2] Buică, A., Françoise, J.P., Llibre J.: Periodic solutions of nonlinear periodic differential systems with a small parameter, Comm. on Pure and Appl. Anal. 6 103-111 (2007)

[3] Caranicolas, N.D.: The structure of motion in a 4-component galaxy mass model. Astrophys. Space Sci. 246, 15-28 (1997)

[4] Caranicolas, N.D.: A new mass model describing motion in axially symmetric galaxies with dark matter. Mon. Not. R. Astron. Soc. 423, 2668-2679 (2012)

[5] Caranicolas N.D., Innanen, K.A.: Chaos in a galaxy model with a nucleus and bulge components. Astron. J. 102, 1343-1347 (1991)

[6] Carlberg, R.G., Innanen, K.A.: Galactic chaos and the circular velocity at the sun. Astron. J. 94, 666-670 (1987)

[7] Cretton, N., de Zeeuw, P.T., van derMarel, R.P., Rix, H.W.: Axisymmetric three-integral models for galaxies. Astrophys. J. Suppl. Ser. 124, 383-401 (1999)

[8] Dehnen, W.D.: A family of potential-density pairs for spherical galaxies and bulges. Mon. Not. R. Astron. Soc. 265, 250-256 (1993)

[9] Malkin, I.G.: Some problems of the theory of nonlinear oscillations, (Russian), Gosudarstv. Izdat. Tehn.Teor. Lit., Moscow, 1956.

[10] Merritt, D., Fridman, T.: Triaxial galaxies with cusps. Astrophys. J. 460, 136-162 (1996)

[11] Miyamoto, M., Nagai, R.: Three-dimensional models for the distribution of mass in galaxies. Publ. Astron. Soc. Jpn. 27, 533-543 (1975)

[12] Rindler-Daller, T., Dejonghe, H., Zeilinger, W.W.: Spherical models for early-type galaxies with cuspy mass densities. Mon. Not. R. Astron. Soc. 356, 1403-1408 (2005) 
[13] Roseau, M.: Vibrations non linéaires et théorie de la stabilité (French), Springer Tracts in Natural Philosophy, Vol. 8 Springer-Verlag, Berlin-New York, 1966.

[14] Sanders, J., Verhulst, F. and Murdock, J. Averaging Methods in Nonlinear Dynamical Systems, Appl. Math. Sci. 59, 2nd. ed., Springer, Berlin, 2007.

[15] Schwarzschild, M.: Self-consistent models for galactic halos. Astrophys. J. 409, 563-577 (1993)

[16] Statler, T.S.: Self-consistent models of perfect triaxial galaxies. Astrophys. J. 321, 113-152 (1987)

[17] Verolme, E.K., de Zeeuw, P.T.: Two-integral Schwarzschild models. Mon. Not. R. Astron. Soc. 331, 959968 (2002)

[18] Zhao, H.S.: Analytical models for galactic nuclei. Mon. Not. R. Astron. Soc. 278, 488-496 (1996)

[19] Zotos, E.E.: A new dynamical model for the study of galactic structure. New Astron. 16, 391-401 (2011)

[20] E.E. Zotos and N.D. Caranicolas, Order and chaos in a new 3D dynamical model describing motion in non-axially symmetric galaxies. Nonlinear Dyn. 74, 1203-1221 (2013)

1 Departament de Matemàtiques, Universitat Autònoma de Barcelona, 08193 Bellaterra, Barcelona, Catalonia, Spain

E-mail address: jllibre@mat.uab.cat

2 Departamento de Matemática, Universidad del Bio-Bio, Concepción, Avda. Collao 1202, Chile

E-mail address: clvidal@ubiobio.cl 